\title{
Adiposity and carotid-intima media thickness in children and adolescents: a systematic review
}

\author{
Min Hae Park ${ }^{1 *}$, Áine Skow ${ }^{1}$, Sara De Matteis ${ }^{1,2}$, Anthony S. Kessel ${ }^{3}$, Sonia Saxena ${ }^{4}$, Russell M. Viner ${ }^{5}$ \\ and Sanjay Kinra ${ }^{1}$
}

\begin{abstract}
Background: Adiposity in childhood is associated with later cardiovascular disease (CVD), but it is unclear whether this relationship is independent of other risk factors experienced in later life, such as smoking and hypertension. Carotid-intima media thickness (CIMT) is a measure of subclinical atherosclerosis that may be used to assess CVD risk in young people. The aim of this study was to examine the relationship between adiposity and cIMT in children and adolescents.

Methods: We searched Medline, Embase, Global Health, and CINAHL Plus electronic databases (1980-2014). Population-based observational studies that reported a measure of association between objectively-measured adiposity and CIMT in childhood were included in this review.

Results: Twenty-two cross-sectional studies were included ( $n=7,366$ children and adolescents). Thirteen of nineteen studies conducted in adolescent populations (mean age $\geq 12$ years, $n=5,986$ ) reported positive associations between CIMT and adiposity measures (correlation coefficients 0.13 to 0.59 ). Three studies of pre-adolescent populations $(n=1,380)$ reported mixed evidence, two studies finding no evidence of a correlation, and one an inverse relationship between skinfolds and cIMT. Included studies did not report an adiposity threshold for subclinical atherosclerosis.

Conclusions: Based on studies conducted mostly in Western Europe and the US, adiposity does not appear to be associated with cIMT in pre-adolescents, but may be associated in adolescents. If further studies confirm these findings, a focus on cardiovascular disease prevention efforts in pre-adolescence, before arterial changes have emerged, may be justified.
\end{abstract}

Keywords: Childhood obesity, Carotid intima-media thickness, Cardiovascular risk

\section{Background}

A number of studies have reported positive associations between body mass index (BMI) in childhood and cardiovascular disease (CVD) risk factors, morbidity and mortality in adulthood [1-4]. However, childhood obesity tracks into adulthood [5], and where studies have been able to account for obesity in adulthood, the associations between childhood obesity and adult cardiovascular disease have been less convincing [6]. This raises questions about the appropriateness of directing cardiovascular disease prevention efforts towards overweight and obese children [7]. Furthermore, many of the other

\footnotetext{
* Correspondence: minhae.park@lshtm.ac.uk

'Department of Non-communicable Disease Epidemiology, London School of Hygiene \& Tropical Medicine, Keppel Street, London WC1E 7HTUK Full list of author information is available at the end of the article
}

conventional risk factors for cardiovascular disease, such as smoking, alcohol intake, high serum cholesterol and blood pressure $[8,9]$, are more prevalent in overweight individuals than their lean counterparts. These other risk factors could explain the observed association between childhood obesity and future cardiovascular disease.

The standard method of accounting for potential alternative explanations in epidemiological studies (i.e. confounding) is to adjust for them in multiple variable regression models. However, this technique is strongly reliant on the accuracy with which these variables can be assessed. The difficulties of accurate variable measurement (e.g. assigning social position or assessing smoking, alcohol or dietary intake accurately from self-reports, and accounting for varying periods of exposure) make adjusted results prone to bias. An alternative analytical approach is 
to restrict the analyses to sub-groups that have little or no exposure to these risk factors. For cardiovascular disease, the prevalence of many of the conventional risk factors such as smoking, excessive alcohol intake, hypercholesterolemia, hypertension or diabetes, is negligible in children and low in adolescents [10-12], making them an ideal group in which to examine the independent contribution of childhood obesity to future CVD risk. Carotid intima media thickness (cIMT) is a measure of subclinical atherosclerosis that is a surrogate for future cardiovascular disease $[13,14]$. A recent systematic review examined the relationship between BMI and CIMT [15], but reported only mean differences in cIMT by categorical weight status rather than the continuous relationships between measures of adiposity and cIMT, and the review was limited to studies in children aged $5-15$ years.

We systematically reviewed the published evidence on the association between measures of adiposity in childhood or adolescence and cIMT. We limited our study to population-based samples, with a view to identifying any thresholds above which the potential effects of adiposity may manifest. We also sought evidence for variations in these associations by age, sex and ethnicity.

\section{Methods}

We searched for published English-language studies of the association between measures of adiposity (including BMI, weight status, body fat percentage, waist circumference) and cIMT (reported as continuous or categorical measures) in childhood and adolescence. Medline, Embase, Global Health, and CINAHL Plus electronic databases were searched (1980-November Week 2, 2014) for relevant publications. Ethical approval was not sought for this study as it was a review of published literature already in the public domain; data were analysed as reported in the original publications.

The following search terms were used for Medline and modified for the other databases: 1. exp Obesity/2. exp Overweight/ 3. exp Body Mass Index/ 4. exp "Body Weights and Measures"/ 5. (obes\$ or obesity).mp. 6. Overweight.mp. 7. (BMI or Body mass index or Body-massindex or Weight for height or Weight-for-height).mp. 8. (Body fat or Body fat percent\$ or Percent\$ body fat or Fat mass or Adiposity).mp. 9. (Waist circumference or Waist measurement).mp. 10. or/1-9. 11. exp Child/ 12. exp Adolescent/13. exp Pediatrics/ 14. juvenile.mp. 15. child\$.mp. 16. adolescen\$.mp. 17. teen\$.mp. 18. P?ediatric\$.mp. 19. or/11-18. 20. exp Carotid Intima-Media Thickness/ 21. (Intima?media thickness or Carotid intima?media thickness or Arterial thickness or Arterial wall thickness).mp. 22. or/20-21. 23. 10 and 19 and 22. 24. Limit 23 to (English language and humans and $\mathrm{yr}=$ "1980 -Current").
Observational studies were eligible for inclusion in the review, while case reports and abstracts were excluded. Studies were included if all of the following criteria were met: (1) they reported on the association between adiposity and cIMT in childhood or adolescence (mean age of population between 2 and 19 years), (2) both adiposity and cIMT were assessed using objective measures, (3) adiposity and cIMT were measured within 24 months of each other, (4) the study was of a community/population-based sample, and (5) sample size $\geq 100$. Studies were excluded if any of the following criteria were met: (1) the study population received an intervention, (2) adiposity was measured in infancy ( $<2$ years), (3) adiposity was assessed based on self- or parent-reported measures, (4) the study population was from an obesity/ specialist clinic or hospital setting, or (5) a participant's inclusion in the study was dependent upon the presence of a secondary comorbidity.

Two independent reviewers (MHP and SDM) screened titles and abstracts for an initial assessment of eligibility. After abstract screening, both reviewers reviewed full text articles to make final decisions on inclusion. Disagreements regarding eligibility were resolved after consultation with a third reviewer (AS).

Data were extracted in duplicate by MHP and SDM using a piloted form. For each study, the following information was recorded: study design, country and dates of study, measures of adiposity and health outcomes used, prevalence of overweight and obesity, characteristics of the study population (sample size, sex, age, ethnicity), cIMT measurement methods, measures of the association between adiposity and cIMT (e.g. regression coefficients, relative risks), and any additional analyses (e.g. adjustment for covariates, stratification by other characteristics). Given the heterogeneity of study populations and measures of effect used, data were synthesised in a narrative fashion.

\section{Results}

Of 676 abstracts screened, 22 studies were eligible for inclusion, representing 7,366 children and adolescents (Fig. 1). Thirteen studies were conducted in Europe [16-28], four in the United States [29-32], two in Turkey [33, 34], and one each in Chile [35], India [36] and South Korea [37]. With the exception of one French study [26], all studies were published within the last decade. Participants ranged in age from 4 to 24 years. Among those studies that reported the figure, the proportion of overweight and obese participants ranged from $11.6 \%$ in Germany [16] to $74.0 \%$ in Turkey [33]. The full descriptions of included studies, including methods used to measure cIMT, are presented in Table 1. The details of studies excluded during the full text review are listed in Additional file 1. 


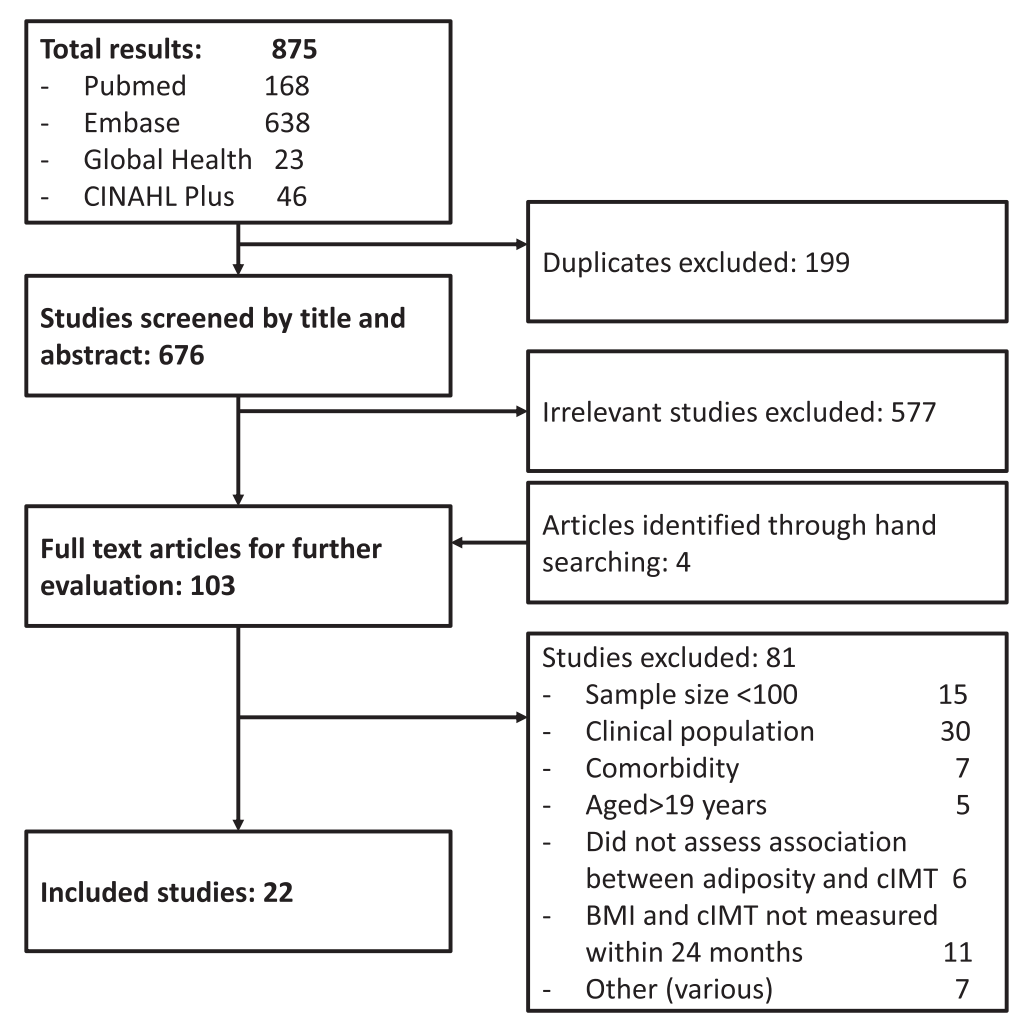

Fig. 1 Flowchart of study selection process during systematic search

\section{The association between adiposity and CIMT}

Nineteen studies reported the relationship between adiposity and cIMT in adolescents or mixed populations of children and adolescents (mean age $\geq 12$ years, $n=5,986$ ). The other three studies were conducted in pre-adolescent populations (mean age $<12$ years, $n=1,380$ ) $[20,25,28]$.

\section{Studies in mixed age or adolescent populations}

Twelve studies of mixed age and adolescent-only samples reported on the correlation between measures of adiposity and cIMT (Table 2). Eight of these (sample sizes ranged from 100 to 1,051) found strong evidence of associations between adiposity measures (BMI, BMISDS, body fat percentage, body fat mass, waist circumference, hip circumference, waist to hip ratio) and cIMT, though in one study [16] the association was observed for girls only. After adjusting for other risk factors in multiple linear regression analyses, measures of adiposity remained an independent predictor of CIMT in six out of seven studies: one study found that BMI SDS was a predictor of age- and height-adjusted cIMT SDS [21]; another reported that after adjusting for ethnicity, systolic blood pressure, HDL-C, LDL-C and total cholesterol/HDL-C ratio, BMI $\mathrm{z}$-score $(\beta=0.008, p<0.0001)$ and waist circumference $(\beta$ $=0.001, p=0.0005)$ were predictive of cIMT in both sexes [31]; a third study showed that waist to hip ratio remained associated with cIMT after adjusting for age, sex, height,
BMI, total cholesterol and blood pressure, but BMI did not [30]; another found BMI SDS to be associated with cIMT after adjusting for systolic blood pressure SDS $(\beta=0.14, p<0.0001)[19]$; in a stepwise multivariable analysis including age, sex, BMI, waist circumference, blood pressure, triglycerides to HDL-C ratio and glucose, waist circumference was found to be a predictor of $\operatorname{cIMT}(\beta=0.001, p<0.01)$, but BMI was not [22]; in the sixth study, after including waist circumference, BMI, body fat mass, trunk fat mass, sex and age in a multivariable model, waist circumference remained associated with $\operatorname{cIMT}(\beta=0.31, p=0.03)$, but the other measures of adiposity did not [24]. The seventh study found that after adjustment for total \% body fat, blood pressure and age, BMI was no longer predictive of cIMT in either sex [16].

The four studies that did not find strong evidence of a correlation between adiposity and cIMT (sample sizes ranged from 193 to 319 ) also assessed a wide range of adiposity measures, including BMI [23, 26, 37], BMI centile [29], fat mass [23, 26], waist circumference [23, 37], waist to hip ratio $[23,26]$ and body fat percentage [26].

Seven additional studies conducted in adolescents examined the relationship between weight category and cIMT values. Five out of these seven studies reported positive associations between weight category and cIMT (Table 3). Caserta et al. [18] reported that the prevalence 
Table 1 Characteristics of studies included in review

\begin{tabular}{|c|c|c|c|c|c|c|}
\hline Reference & Country & Population (n) & $\begin{array}{l}\% \text { overweight } \\
\text { (incl. obese) }\end{array}$ & $\%$ female & $\begin{array}{l}\text { Age range, } \\
\text { years }\end{array}$ & cIMT measurement method \\
\hline $\begin{array}{l}\text { Arnaiz et al. } \\
2010 \text { [35] }\end{array}$ & Chile & Schools (103) & 56 & 46.6 & $6-16$ & $\begin{array}{l}\text { Ultrasonography; measurement location } \\
\text { not reported; automated edge detection. } \\
\text { Number of measurements not reported. }\end{array}$ \\
\hline $\begin{array}{l}\text { Böhm et al. } \\
2009 \text { [16] }\end{array}$ & Germany & Schools (267) & 11.6 & 53.6 & $6-17$ & $\begin{array}{l}\text { Ultrasonography; measurement proximal to } \\
\text { carotid artery bifurcation; automated edge } \\
\text { detection. Mean of } 11 \text { measurements used } \\
\text { for analysis. }\end{array}$ \\
\hline $\begin{array}{l}\text { Casariu et al. } \\
2011 \text { [17] }\end{array}$ & Romania & Schools (100) & 50 & 58.0 & $6-18$ & $\begin{array}{l}\text { Ultrasonography; measurement at the common } \\
\text { carotid artery near the bifurcation, during end } \\
\text { diastole. Maximal thicknesses of the intima-media } \\
\text { width measured to give three readings and the } \\
\text { mean value was used for analysis. }\end{array}$ \\
\hline $\begin{array}{l}\text { Caserta et al. } \\
2010 \text { [18] }\end{array}$ & Italy & Primary schools (575) & 31.1 & 49.9 & $11-13$ & $\begin{array}{l}\text { Ultrasonography; measurement of far wall at } 3 \\
\text { locations below bifurcation. Mean of these } \\
\text { measurements used for analysis. }\end{array}$ \\
\hline $\begin{array}{l}\text { Croymans et al. } \\
2010 \text { [29] }\end{array}$ & United States & High schools (249) & $\begin{array}{l}15 \% \text { had BMl } \\
\text { centile > 95th }\end{array}$ & 67.5 & $15-18$ & $\begin{array}{l}\text { Ultrasonography; images of the far wall of the } \\
\text { common carotid artery taken from multiple } \\
\text { angles; automated edge detection. Mean of } \\
\text { these measurements used for analysis. }\end{array}$ \\
\hline
\end{tabular}

$\begin{array}{lllll}\begin{array}{l}\text { Dawson et al. } \\ 2009[30]\end{array} & \text { United States } & \begin{array}{l}\text { Offspring of } \\ \text { population-based } \\ \text { cohort }(228 \text { aged }<18)\end{array} & 44.3 & 11-17\end{array}$

Ultrasonography; near and far wall of the left and right internal, bifurcation and common carotid arteries imaged at three angles; automated edge detection. The mean across angles used to obtain location-specific means; the average of these 12 measures used for analysis.

\begin{tabular}{|c|c|c|c|}
\hline $\begin{array}{l}\text { Doyon et al. } \\
2013 \text { [19] }\end{array}$ & $\begin{array}{l}\text { Turkey, Germany, } \\
\text { Sweden, Poland }\end{array}$ & $\begin{array}{l}\text { Nonobese, } \\
\text { nonhypertensive } \\
\text { children }(1,051)\end{array}$ & $\begin{array}{l}\text { Nonobese } \\
\text { sample }\end{array}$ \\
\hline
\end{tabular}

\begin{tabular}{|c|c|c|c|c|c|}
\hline $\begin{array}{l}\text { Elkiran } \\
2013 \text { [33] }\end{array}$ & Turkey & Schools (123) & 74.0 & 54.5 & $11-15$ \\
\hline $\begin{array}{l}\text { Geerts } \\
2012 \text { [20] }\end{array}$ & Netherlands & $\begin{array}{l}\text { Population-based } \\
\text { birth cohort (306) }\end{array}$ & NR & 55.5 & 5 \\
\hline $\begin{array}{l}\text { Jourdan et al. } \\
2005 \text { [21] }\end{array}$ & $\begin{array}{l}\text { Germany and } \\
\text { Poland }\end{array}$ & Schools (247) & NR & 51.4 & $10-20$ \\
\hline $\begin{array}{l}\text { Kollias et al. } \\
2013 \text { [22] }\end{array}$ & Greece & Schools (448) & $\begin{array}{l}28.1 \% \\
\text { overweight, } \\
12.7 \% \text { obese }\end{array}$ & 52.9 & $10-18$ \\
\hline
\end{tabular}

\begin{tabular}{|c|c|c|c|c|}
\hline $\begin{array}{l}\text { Lamotte et al. } \\
2013 \text { [23] }\end{array}$ & France & Schools (319) & $\begin{array}{l}13.5 \% \\
\text { overweight } \\
3.4 \% \text { obese }\end{array}$ & $57.7 \%$ \\
\hline
\end{tabular}

Ultrasonography; CIMT was obtained either by 5 averaged measurements on each common carotid artery or semiautomatically using a digital image evaluation software, depending on the availability of the software package at each centre.

Ultrasonography; measurement of left common carotid artery. Number of measurements not reported.
Ultrasonography (high resolution echo-tracking); measurement of right common carotid artery. Measurement repeated a maximum of four times.

Ultrasonography; far wall measured manually using the calliper method.

Ultrasonography; bilateral measurements at the point of maximum thickness on the far wall along a $1 \mathrm{~cm}$ section of each common carotid artery proximal to the carotid bulb; measurement using electronic calipers. Mean of 3-4 measurements for each side used for analysis.

Ultrasonography; bilateral assessment along $10 \mathrm{~mm}$ segment of common carotid arteries, $\geq 5 \mathrm{~mm}$ from the bifurcation; one hundred measurements on average on the far wall on each side; automated. Mean value of left and right measures used for analysis.

$\begin{array}{llllll}\text { Lim et al. } & \text { South Korea } & \text { High school (285) } & 13.3 & 48.4 & 14-17 \\ 2009 \text { [37] } & & & & \\ & & & & \\ \begin{array}{l}\text { Melo et al. } \\ 2014 \text { [24] }\end{array} & \text { Portugal } & \text { Schools (385) } & 28.3 & 50.9 & 11-13\end{array}$

Ultrasonography; bilateral measurements of near and far walls of common carotid arteries; Automated edge-detection. Maximum IMT value determined for each side and the average used for analysis. 
Table 1 Characteristics of studies included in review (Continued)

\begin{tabular}{|c|c|c|c|c|c|c|}
\hline $\begin{array}{l}\text { Mittelman et al. } \\
2010 \text { [31] }\end{array}$ & United States & $\begin{array}{l}\text { Schools and } \\
\text { universities (599) }\end{array}$ & 32.7 & 51.3 & $6-20$ & $\begin{array}{l}\text { Ultrasonography; measurement on far walls of } \\
\text { left common carotid artery } 1 \mathrm{~cm} \text { proximal to the } \\
\text { bifurcation during } 3 \text { complete separate cardiac } \\
\text { cycles; Automated edge-detection. Average } \\
\text { reading of all } 3 \text { systolic and } 3 \text { diastolic frames } \\
\text { used for analyses. }\end{array}$ \\
\hline $\begin{array}{l}\text { Osiniri et al. } \\
\text { [25] } 2012\end{array}$ & Spain & $\begin{array}{l}\text { Well child visits at } \\
\text { primary care } \\
\text { centres (135) }\end{array}$ & NR & 53.3 & $\begin{array}{l}\mathrm{NR} \text {, mean } \\
7.1 \pm 1.1\end{array}$ & $\begin{array}{l}\text { Ultrasonography; diastolic images obtained from } \\
\text { far wall of the distal common carotid artery } 1 \mathrm{~cm} \\
\text { from bifurcation. Averages of } 5 \text { measurements } \\
\text { used for analyses. }\end{array}$ \\
\hline $\begin{array}{l}\text { Ozguven et al. } \\
2010 \text { [34] }\end{array}$ & Turkey & Schools (142) & 49.3 & 55.6 & $13-18$ & $\begin{array}{l}\text { Ultrasonography; measurement on far wall of left } \\
\text { common carotid artery. Mean of at least four } \\
\text { measurements taken } \sim 10 \mathrm{~mm} \text { proximal to } \\
\text { bifurcation used for analyses. }\end{array}$ \\
\hline $\begin{array}{l}\text { Pandit et al. } \\
2014[36]\end{array}$ & India & $\begin{array}{l}\text { Private schools; } \\
\text { routine health } \\
\text { checks }(250)\end{array}$ & 71.2 & NR & $6-17$ & $\begin{array}{l}\text { Ultrasonography (echo-tracking); measurement } \\
\text { at right common carotid artery; Automated } \\
\text { edge-detection. Number of measurements not } \\
\text { reported. }\end{array}$ \\
\hline $\begin{array}{l}\text { Sass et al. } \\
1998 \text { [26] }\end{array}$ & France & $\begin{array}{l}\text { Population-based } \\
\text { cohort (193) }\end{array}$ & NR & 56.0 & $\begin{array}{l}\text { 10-24 } \\
\text { (mean 15.5) }\end{array}$ & $\begin{array}{l}\text { Ultrasonography; bilateral measurement } \\
\text { on } \geq 1 \mathrm{~cm} \text { segment of carotid arteries at } 3 \mathrm{~cm} \\
\text { proximal to the bifurcation. Average of } 25-50 \\
\text { readings per measurement, with two } \\
\text { measurements obtained per segment. Average of } \\
\text { right and left measurements used for analysis. }\end{array}$ \\
\hline $\begin{array}{l}\text { Urbina et al. } \\
2009 \text { [32] }\end{array}$ & United States & 318 & 43.4 & 62.3 & $\begin{array}{l}\text { 10-24 (mean } \\
\text { age 17.8) }\end{array}$ & $\begin{array}{l}\text { Ultrasonography; bilateral measurements of three } \\
\text { segments of carotid arteries. Trace technique to } \\
\text { measure maximum thickness on right and left } \\
\text { sides, and averaged for the common carotid } \\
\text { artery, the bifurcation (carotid bulb), and the } \\
\text { internal carotid artery. }\end{array}$ \\
\hline $\begin{array}{l}\text { Weghuber et al. } \\
2013 \text { [27] }\end{array}$ & Austria & $\begin{array}{l}\text { Residents of Graz and } \\
\text { Styria (104 subsample) }\end{array}$ & 46.2 & 56.7 & $4-18$ & $\begin{array}{l}\text { Ultrasonography; bilateral measurements of the } \\
\text { bulbous near common carotid arteries. Maximal } \\
\text { IMT recorded at each of the vessel segments } \\
\text { and averaged for each side. }\end{array}$ \\
\hline $\begin{array}{l}\text { Whincup et al. } \\
2012 \text { [28] }\end{array}$ & United Kingdom & Primary schools (939) & NR & 53 & $\begin{array}{l}\text { NR, mean } \\
10.8 \pm 0.4\end{array}$ & $\begin{array}{l}\text { Ultrasonography; bilateral measurement on far } \\
\text { walls of common carotid arteries proximal to } \\
\text { the carotid bifurcation. Three end-diastolic } \\
\text { frames selected and analyzed for mean cIMT } \\
\text { on each side. Mean of left- and right-sided } \\
\text { readings used for analysis. }\end{array}$ \\
\hline
\end{tabular}

of abnormal cIMT (defined as values above the 75th centile of the study population) was higher in obese adolescents than non-overweight adolescents $(41.4 \%$ versus $20.7 \%$ among females, $43.4 \%$ versus $28.3 \%$ among males). Four studies reported that mean cIMT was higher in overweight and obese children than in normal weight children (Table 3) [27, 33, 34, 36], with the difference in cIMT between obese and normal weight groups ranging from $0.03 \mathrm{~mm}$ [36] to $0.2 \mathrm{~mm}$ [27].

\section{Studies in pre-adolescent populations}

Three studies of pre-adolescents (mean age $<12$ years) did not find evidence of a positive association between cIMT and adiposity [20, 25, 28]. The largest of these was a UK study of school children with a mean age of 10.8 years $(n=939)$, which reported the absolute difference in cIMT for a 1 standard deviation increase in adiposity measures of ponderal index, fat mass index, and sum of skinfolds [28]; after adjusting for age, sex, ethnicity, observer, and month, there was no association between CIMT and either ponderal index or fat mass index, but sum of skinfolds was inversely associated with cIMT (Table 3). A Spanish study [25] found that in children aged 6-8 years, there was no correlation between cIMT and body fat percentage, BMI z-score, waist circumference, or visceral fat (Table 2). Similarly, a Dutch study of children aged 5 years showed that there was no association between BMI tertiles and cIMT [20].

\section{Factors affecting the association between adiposity and CIMT}

Four studies presented results from analyses stratified by sex $[16,23,26,31]$. In univariate analyses, Böhm et al. [16] observed a stronger correlation between cIMT and 
Table 2 Associations between adiposity and CIMT in children and adolescents, expressed as correlation coefficients

\begin{tabular}{|c|c|c|c|c|c|c|c|c|}
\hline \multirow[t]{2}{*}{ Reference } & \multirow{2}{*}{$\begin{array}{l}\text { Age range, } \\
\text { years }\end{array}$} & \multirow[t]{2}{*}{ Adiposity measure } & \multicolumn{2}{|c|}{ All participants } & \multicolumn{2}{|c|}{ Females } & \multicolumn{2}{|l|}{ Males } \\
\hline & & & $r$ & $\mathrm{P}$ & $r$ & $\mathrm{P}$ & $r$ & $\mathrm{P}$ \\
\hline \multicolumn{9}{|c|}{ Studies in mixed age or adolescent populations (mean age $\geq 12$ years) } \\
\hline \multirow[t]{2}{*}{ Böhm et al. 2009 [16] } & $6-17$ & $\mathrm{BMl}$ & - & - & 0.35 & $<0.001$ & 0.14 & 0.11 \\
\hline & & Body fat $\%$ & - & - & 0.41 & $<0.001$ & 0.06 & 0.46 \\
\hline \multirow[t]{3}{*}{ Casariu et al. 2011 [17] } & $6-18$ & $\mathrm{BMl}$ & 0.49 & $<0.05$ & - & - & - & - \\
\hline & & Waist circumference & 0.59 & $<0.05$ & - & - & - & - \\
\hline & & Hip circumference & 0.58 & $<0.05$ & - & - & - & - \\
\hline Croymans et al. 2010 [29] & $15-18$ & BMI centile & $0.08^{*}$ & $0.22^{*}$ & - & - & - & - \\
\hline \multirow[t]{2}{*}{ Dawson et al. 2009 [30] } & $11-17$ & $\mathrm{BMl}$ & 0.173 & $<0.05$ & - & - & - & - \\
\hline & & Waist/hip ratio & 0.310 & $<0.001$ & - & - & - & - \\
\hline \multirow[t]{2}{*}{ Doyon et al. 2013 [19] } & $6-18$ & $\mathrm{BMl}$ & 0.13 & $<0.001$ & - & - & - & - \\
\hline & & BMI SDS & 0.13 & $<0.001$ & - & - & - & - \\
\hline \multirow[t]{2}{*}{ Jourdan et al. 2005 [21] } & $10-20$ & $\mathrm{BMI}$ & 0.25 & $<0.001$ & - & - & - & - \\
\hline & & $\mathrm{BMI} \mathrm{SDS}^{\mathrm{a}}$ & 0.41 & $<0.001$ & - & - & - & - \\
\hline \multirow[t]{3}{*}{ Kollias et al. 2013 [22] } & $10-18$ & BMI Z-score & 0.05 & NS & - & - & - & - \\
\hline & & Waist circumference & 0.12 & $<0.05$ & - & - & - & - \\
\hline & & Waist to hip ratio & 0.12 & $<0.05$ & - & - & - & - \\
\hline \multirow[t]{5}{*}{ Lamotte et al. 2013 [23] } & $12-17$ & $\mathrm{BMl}$ & 0.03 & NS & -0.03 & NS & 0.09 & NS \\
\hline & & BMI Z-score & 0.04 & NS & -0.002 & NS & 0.08 & NS \\
\hline & & Fat mass & -0.04 & NS & -0.06 & NS & 0.04 & NS \\
\hline & & Waist circumference & 0.04 & NS & -0.07 & NS & 0.1 & NS \\
\hline & & Waist/hip ratio & 0.04 & NS & -0.03 & NS & 0.02 & NS \\
\hline \multirow[t]{2}{*}{ Lim et al. 2009 [37] } & $14-17$ & $\mathrm{BMI}$ & 0.0499 & 0.402 & - & - & - & - \\
\hline & & Waist circumference & -0.0215 & 0.718 & - & - & - & - \\
\hline \multirow[t]{4}{*}{ Melo et al. 2014 [24] } & $11-13$ & BMl & 0.188 & $<0.001$ & - & - & - & - \\
\hline & & Body fat mass by DXA & 0.153 & 0.003 & - & - & - & - \\
\hline & & Trunk fat mass by DXA & 0.155 & 0.002 & - & - & - & - \\
\hline & & Waist circumference & 0.221 & $<0.001$ & - & - & - & - \\
\hline \multirow[t]{4}{*}{ Mittelman et al. 2010 [31] } & $6-20$ & BMl z-score & - & - & 0.34 & $<0.001$ & 0.30 & $<0.001$ \\
\hline & & Waist circumference & - & - & 0.33 & $<0.001$ & 0.32 & $<0.001$ \\
\hline & & Hip circumference & - & - & 0.27 & $<0.001$ & 0.26 & $<0.001$ \\
\hline & & Waist/hip ratio & - & - & 0.20 & 0.005 & 0.13 & 0.0271 \\
\hline \multirow[t]{4}{*}{ Sass et al. 1998 [26] } & $10-24$ & Fat mass $(\mathrm{kg})$ & - & - & -0.029 & NS & 0.135 & NS \\
\hline & & Body fat $\%$ & - & - & -0.057 & NS & 0.024 & NS \\
\hline & & $\mathrm{BMl}$ & - & - & -0.033 & NS & 0.146 & NS \\
\hline & & Waist/hip ratio & - & - & 0.069 & NS & -0.016 & NS \\
\hline \multicolumn{9}{|c|}{ Studies in pre-adolescent populations (mean age $<12$ years) } \\
\hline \multirow[t]{3}{*}{ Osiniri et al. 2012 [25] } & Mean $7.1 \pm 1.1$ & BMl z-score ${ }^{b}$ & 0.040 & NS & - & - & - & - \\
\hline & & Body fat $\%$ & 0.042 & NS & - & - & - & - \\
\hline & & waist circumference ${ }^{b}$ & 0.048 & NS & - & - & - & - \\
\hline
\end{tabular}

Coefficients in bold were statistically significant $(P<0.05)$

CIMT carotid intima-media thickness, BMI body mass index $\mathrm{kg} / \mathrm{m}^{2}, D X A$ Dual-energy X-ray absorptiometry, NS not significant, $P$-value not reported, SDS standard deviation score

${ }^{*} r$ and $P$ values obtained from the author

${ }^{a}$ Outcome was age- and height-specific cIMT standard deviation score

${ }^{b}$ Log transformed 
Table 3 Other measures of associations between cIMT and adiposity measures in children and adolescents

\begin{tabular}{|c|c|c|c|c|c|}
\hline Reference & Age range, years & Adiposity measure(s) & \multicolumn{2}{|l|}{ Measure of association } & $P$ value \\
\hline \multicolumn{6}{|c|}{ Studies in mixed age or adolescent populations (mean age $\geq 12$ years) } \\
\hline & & & \multicolumn{2}{|l|}{ Mean cIMT (mm) } & \\
\hline \multirow[t]{3}{*}{ Arnaiz 2010 [35] } & $6-16$ & Normal weight & \multicolumn{2}{|l|}{$0.50 \pm 0.03$} & NR \\
\hline & & Overweight & \multicolumn{2}{|l|}{$0.49 \pm 0.02$} & \\
\hline & & Obese & \multicolumn{2}{|l|}{$0.49 \pm 0.03$} & \\
\hline \multirow[t]{3}{*}{ Elkiran 2013 [33] } & $11-15$ & Healthy weight & \multicolumn{2}{|l|}{$0.36 \pm 0.009$} & 0.001 \\
\hline & & Overweight & \multicolumn{2}{|l|}{$0.52 \pm 0.008$} & \\
\hline & & Obese & \multicolumn{2}{|l|}{$0.53 \pm 0.008^{\complement}$} & \\
\hline \multirow[t]{3}{*}{ Ozguven et al. 2010 [34] } & $13-18$ & Normal weight & \multicolumn{2}{|l|}{$0.51 \pm 0.005$} & $<0.001$ \\
\hline & & Overweight & \multicolumn{2}{|l|}{$0.57 \pm 0.009$} & \\
\hline & & Obese & \multicolumn{2}{|l|}{$0.64 \pm 0.007^{d}$} & \\
\hline \multirow[t]{2}{*}{ Pandit et al. 2014 [36] } & $6-17$ & Normal weight & \multicolumn{2}{|l|}{$0.31 \pm 0.01$} & $<0.05$ \\
\hline & & Overweight/obese & \multicolumn{2}{|l|}{$0.34 \pm 0.01$} & \\
\hline \multirow[t]{2}{*}{ Urbina et al. 2009 [32] } & $10-24$ & Lean & \multicolumn{2}{|l|}{$0.52 \pm 0.08$} & NS \\
\hline & & Obese & \multicolumn{2}{|l|}{$0.50 \pm 0.09$} & \\
\hline \multirow[t]{3}{*}{ Weghuber et al. 2013} & $4-18$ & Normal weight & \multicolumn{2}{|l|}{$0.5(95 \% \mathrm{Cl} 0.4$ to 0.6$)$} & $<0.001$ \\
\hline & & Overweight/obese & \multicolumn{2}{|l|}{0.7 (95\% Cl 0.6 to 0.7$)$} & \\
\hline & & & \multicolumn{2}{|l|}{ clMT > 75th centile (\%) } & \\
\hline \multirow[t]{4}{*}{ Caserta et al. 2010 [18] } & $11-13$ & & Males & Females & \\
\hline & & Non-overweight & $28.3 \%$ & $20.7 \%$ & $<0.05$ \\
\hline & & Overweight & $38.9 \%$ & $34.8 \%^{a}$ & \\
\hline & & Obese & $43.4 \%^{a}$ & $41.4 \%^{a, b}$ & \\
\hline
\end{tabular}

Studies in pre-adolescent populations (mean age $<12$ years)

Mean cIMT $(\mu \mathrm{m})$

\begin{tabular}{|c|c|c|c|}
\hline \multirow[t]{5}{*}{ Geerts et al. 2012 [20] } & \multirow[t]{5}{*}{5} & \multicolumn{2}{|l|}{ BMI tertiles } \\
\hline & & First & $380.4 \pm 37.4$ \\
\hline & & Second & $387.4 \pm 32.8$ \\
\hline & & Third & $389.9 \pm 41.0$ \\
\hline & & & Change in CIMT $(\mathrm{mm})$ per SD increase of adiposity $r$ \\
\hline \multirow[t]{3}{*}{ Whincup et al. 2012 [28] } & \multirow[t]{3}{*}{ NR, mean $10.8 \pm 0.4$} & Ponderal index ${ }^{a}$ & $-0.0007(-0.0029$ to 0.0015$)$ \\
\hline & & Skinfolds ${ }^{a}$ & $-0.0026(-0.0048$ to -0.0004$)$ \\
\hline & & Fat mass index ${ }^{a}$ & $-0.0019(-0.0041$ to 0.0003$)$ \\
\hline
\end{tabular}

each adiposity measure (BMI and body fat \%) in females than in males (Table 2), though these effects were attenuated to the null in multivariable analyses. In their analysis of a large US sample $(n=599)$, Mittelman et al. [31] found no difference in the effect of adiposity on cIMT by sex: the correlation coefficients for all reported adiposity measures were similar for males and females (Table 2). The other two studies reported negative correlation coefficients in girls and positive coefficients in boys, but none of these effects was statistically significant $[23,26]$. None of the studies included in this review reported the relationship between adiposity and cIMT by ethnicity or age.

\section{Discussion}

This review has shown there is a growing body of evidence for a positive relationship between adiposity and cIMT in adolescents, but not in younger children. The 
studies we included did not identify a threshold level of adiposity that led to increased cIMT, and there was little information available on whether the association between adiposity and cIMT varied by characteristics such as ethnicity, age or lifestyle behaviors.

Thirteen out of nineteen studies of adolescent populations (mean age $\geq 12$ years) reported positive associations between cIMT and adiposity measures, including measures of weight-for-height (BMI), body composition (fat mass, body fat percentage), and fat distribution (waist and hip circumferences and their ratio). Comparing studies that found an association between adiposity and cIMT with studies that did not, there was no clear pattern according to the measures of adiposity used or study location, but there may have been differences in sample characteristics, notably the prevalence of overweight and obesity. Three of the four studies that did not find evidence of a correlation between adiposity and cIMT were conducted in relatively lean populations in France [23, 26] and South Korea [37], with prevalence of overweight and obesity $<17 \%$, compared to $30-60 \%$ in the majority of studies that showed a positive correlation between adiposity and cIMT, although positive correlations were also found in samples with low prevalence of overweight [16] and no obese participants [19]. The fourth study examined data from three diverse school populations: a predominantly Hispanic $(94 \%)$ and female (78 \%) student body, a mixed-ethnicity school, and a conservative religious school (Seventh Day Adventist [SDA]) with a majority of female (72\%) students [29]. Pooling the results from these populations may have masked important differences in risk profiles, as SDA students were significantly leaner than other students and Hispanic students had significantly lower mean cIMT values. Analyses were not stratified by school, therefore the potential impact of ethnicity or lifestyle (Seventh Day Adventists follow a primarily vegetarian diet and abstain from alcohol, tobacco, and caffeinated drinks [38]) on the effect of adiposity on cIMT could not be assessed.

Of the three studies of younger, pre-adolescent children, a large UK study of children with mean age 10.8 years reported mixed associations between cIMT and three measures of adiposity [28], while studies in younger Spanish and Dutch children found no strong evidence of an association [20,25]. These results, when considered together with the other findings of this review, suggest that thickening of the carotid artery with adiposity may only become detectable in later childhood and adolescence. This is consistent with the age-related transition from aortic fatty streaks to atherosclerotic lesions [39], and could also indicate that exposure to excess adiposity (or some other risk factor associated with overweight) accelerates these age-related arterial changes
[40]. Recent evidence suggests that duration, more than degree, of obesity is an important factor in the onset of CVD [41]. It may therefore be the case that a minimum duration of exposure to overweight is needed before atherosclerosis is detectable, and this is unlikely to occur in pre-adolescence. Another possible explanation is that metabolic complications associated with obesity such as insulin resistance, which become manifest with longer exposure to excess weight, may explain changes in cIMT rather than adiposity itself [42]. The physiological processes underlying the observed association in adolescents could not be ascertained from this review, but disentangling the independent and synergistic effects of age, obesity and other risk factors should be a focus of future research.

The studies included in our review reported correlation coefficients that suggest that the association between adiposity and cIMT is linear, though few studies explicitly characterized the nature of the relationship. None of the studies reported a weight threshold for atherosclerosis at the studied levels of BMI; most of the studies were conducted in high income, western populations with relatively high prevalence of childhood overweight, therefore potential threshold effects at high levels of adiposity could have been assessed. A handful of studies conducted in populations with low prevalence of overweight and obesity did not examine threshold effects at low levels of adiposity. Further studies in populations that cover a wider range of BMI may be informative, as would those that describe in greater detail the nature (shape) of the association between adiposity and cIMT in young people. Studies that assess the effect of other risk factors on the relationship between adiposity and cIMT are also lacking; such studies may help to identify subgroups of the population that are at increased risk of arterial changes at any given level of adiposity, who may be targets for cardiovascular disease prevention interventions.

Our review expands on the findings of a recent metaanalysis, which reported that obese children have higher mean cIMT than non-obese children [15]. The metaanalysis included five studies, including two which we excluded because they were conducted in clinic-based populations [43, 44]. Our review included several recent studies that were published since the meta-analysis was conducted, and also included studies covering a wider age range (the meta-analysis included study populations aged 5 to 15 years), which enabled disaggregated assessment of studies conducted in pre- and post-adolescent populations.

One limitation of this review is that the finding of no association between adiposity and cIMT in younger children is based on only three studies. Furthermore, a meta-analysis of results was not conducted due to the 
heterogeneity of populations and adiposity measures used in the included studies. Additionally, we chose to use cIMT as a proxy for CVD risk in young people, despite the fact that there are few cardiovascular events in this age group. Also, variations in cIMT measurement methods may have affected our results, including the number and location of measurements, and whether measurements were manual or automated.

\section{Conclusions}

Based on studies conducted mostly in Western Europe and the US, our review has shown that adiposity is positively correlated with cIMT in adolescents, but not in younger children. Studies are needed to confirm these findings, but if these relationships are consistently demonstrated in future research there may be justification for cardiovascular disease prevention efforts in overweight children that begin before adolescence, when arterial changes have yet to emerge.

\section{Availability of supporting data}

For information about access to data extraction forms researchers should contact the corresponding author.

\section{Additional files}

Additional file 1: Table of studies excluded from review. (PDF $221 \mathrm{~kb}$ ) Additional file 2: PRISMA checklist. (DOC $63 \mathrm{~kb}$ )

\section{Abbreviations}

BMI SDS: Body mass index standard deviation score; CIMT: Carotid-intima media thickness; CVD: Cardiovascular disease.

\section{Competing interests}

ASK is also Director of Public Health Strategy and Director of Research and Development at Public Health England (PHE). The views expressed in this paper are those of the authors and are not intended to represent the views of PHE. The other authors have no conflicts of interest relevant to this article to disclose.

\section{Authors' contributions}

MHP contributed to the literature search, study screening, data extraction, data synthesis and interpretation, participated in drafting the manuscript, and approved the final manuscript. ÁS contributed to study screening, data extraction, data synthesis and interpretation, participated in drafting the manuscript, and approved the final manuscript. SDM contributed to the literature search, study screening and data extraction, and critically reviewed and approved the final manuscript. ASK contributed to interpreting the data, critically reviewed and approved the final manuscript. SS contributed to interpreting the data, critically reviewed and approved the final manuscript. RMV contributed to interpreting the data, critically reviewed and approved the final manuscript. SK conceptualized the review, advised on data synthesis and interpretation, critically reviewed the manuscript, and approved the final manuscript.

\section{Acknowledgements}

This article presents independent research funded by the National Institute for Health Research (NIHR) in England under its Programme Grants for Applied Research programme (RP-PG-0608-10035 - The Paediatric Research in Obesity Multi-modal Intervention and Service Evaluation (PROMISE) programme). The views expressed in this publication are those of the authors and do not necessarily reflect those of the NHS, the NIHR, or the
Department of Health. SS is funded by an NIHR Career Development Fellowship. The funder had no role in the design, collection, analysis or interpretation of data, in the writing of the manuscript or the decision to submit the manuscript for publication.

\section{Author details}

${ }^{1}$ Department of Non-communicable Disease Epidemiology, London School of Hygiene \& Tropical Medicine, Keppel Street, London WC1E 7HTUK. ${ }^{2}$ Department of Respiratory Epidemiology, Occupational Medicine and Public Health, NHLI Imperial College London, London, UK. ${ }^{3}$ Faculty of Public Health and Policy, London School of Hygiene \& Tropical Medicine, London, UK. ${ }^{4}$ Department of Primary Care and Public Health, Imperial College London, London, UK. ${ }^{5}$ Department of General and Adolescent Paediatrics, Institute of Child Health, University College London, London, UK.

Received: 5 August 2014 Accepted: 6 October 2015

Published online: 16 October 2015

\section{References}

1. Falkstedt D, Hemmingsson T, Rasmussen F, Lundberg I. Body mass index in late adolescence and its association with coronary heart disease and stroke in middle age among Swedish men. Int J Obes (Lond). 2007;31:777-83.

2. Must A, Jacques PF, Dallal GE, Bajema CJ, Dietz WH. Long-term morbidity and mortality of overweight adolescents. A follow-up of the Harvard Growth Study of 1922 to 1935. N Engl J Med. 1992;327:1350-5.

3. Silventoinen K, Magnusson PK, Tynelius P, Batty GD, Rasmussen F. Association of body size and muscle strength with incidence of coronary heart disease and cerebrovascular diseases: a population-based cohort study of one million Swedish men. Int J Epidemiol. 2009;38:110-8.

4. Tirosh A, Shai I, Afek A, Dubnov-Raz G, Ayalon N, Gordon B, et al. Adolescent BMI trajectory and risk of diabetes versus coronary disease. N Engl J Med. 2011;364:1315-25.

5. Singh AS, Mulder C, Twisk JW, van Mechelen W, Chinapaw MJ. Tracking of childhood overweight into adulthood: a systematic review of the literature. Obes Res. 2008;9:474-88.

6. Park MH, Falconer C, Viner RM, Kinra S. The impact of childhood obesity on morbidity and mortality in adulthood: a systematic review. Obes Rev. 2012;13:985-1000

7. Kavey R-EW, Daniels SR, Lauer RM, Atkins DL, Hayman LL, Taubert K. American heart association guidelines for primary prevention of atherosclerotic cardiovascular disease beginning in childhood. Circulation. 2003;107:1562-6.

8. Lahti-Koski M, Pietinen P, Heliovaara M, Vartiainen E. Associations of body mass index and obesity with physical activity, food choices, alcohol intake, and smoking in the 1982-1997 FINRISK Studies. Am J Clin Nutr. 2002;75:809-17.

9. Malnick SDH, Knobler H. The medical complications of obesity. QJM. 2006;99:565-79.

10. Salonen R, Salonen JT. Determinants of carotid intima-media thickness: a population-based ultrasonography study in eastern Finnish men. J Intern Med. 1991;229:225-31.

11. Tell GS, Howard G, McKinney WM. Risk factors for site specific extracranial carotid artery plaque distribution as measured by B-mode ultrasound. J Clin Epidemiol. 1989:42:551-9.

12. Whisnant JP, Homer D, Ingall TJ, Baker Jr HL, O'Fallon WM, Wievers DO Duration of cigarette smoking is the strongest predictor of severe extracranial carotid artery atherosclerosis. Stroke. 1990;21:707-14.

13. Lorenz MW, Markus HS, Bots ML, Rosvall M, Sitzer M. Prediction of clinical cardiovascular events with carotid intima-media thickness: a systematic review and meta-analysis. Circulation. 2007;115:459-67.

14. Peters SA, Grobbee DE, Bots ML. Carotid intima-media thickness: a suitable alternative for cardiovascular risk as outcome? Eur J Cardiovasc Prev Rehabil. 2011;18:167-74.

15. Friedemann C, Heneghan C, Mahtani K, Thompson M, Perera R, Ward AM. Cardiovascular disease risk in healthy children and its association with body mass index: systematic review and meta-analysis. BMJ. 2012;345, e4759.

16. Bohm B, Hartmann K, Buck M, Oberhoffer R. Sex differences of carotid intima-media thickness in healthy children and adolescents. Atherosclerosis. 2009;206:458-63. 
17. Casariu ED, Virgolici B, Greabu M, Totan A, Daniela M, Mitrea N, et al. Associations between carotid intimamedia thickness and cardiovascular risk markers in obese children. Farmacia. 2011;59:471-82.

18. Caserta CA, Pendino GM, Alicante S, Amante A, Amato F, Fiorillo M, et al. Body mass index, cardiovascular risk factors, and carotid intima-media thickness in a pediatric population in Southern Italy. J Pediatr Gastroentero Nutr. 2010;51:216-20

19. Doyon A, Kracht D, Bayazit AK, Deveci M, Duzova A, Krmar RT, et al. Carotid artery intima-media thickness and distensibility in children and adolescents: Reference values and role of body dimensions. Hypertension. 2013:62:550-6.

20. Geerts CC, Evelein AM, Bots ML, van der Ent CK, Grobbee DE, Uiterwaal CS. Body fat distribution and early arterial changes in healthy 5-year-old children. Ann Med. 2012:44:350-9.

21. Jourdan C, Wühl E, Litwin M, Fahr K, Trelewicz J, Jobs K, et al. Normative values for intima-media thickness and distensibility of large arteries in healthy adolescents. J Hypertens. 2005;23:1707-15.

22. Kollias A, Psilopatis I, Karagiaouri E, Glaraki M, Grammatikos E, Grammatikos $\mathrm{EE}$, et al. Adiposity, blood pressure, and carotid intima-media thickness in greek adolescents. Obesity (Silver Spring). 2013;21:1013-7.

23. Lamotte C, Iliescu C, Beghin L, Salleron J, Gonzalez-Gross M, Marcos A, et al. Association of socioeconomic status, truncal fat and sICAM-1 with carotid intima-media thickness in adolescents: the HELENA study. Atherosclerosis. 2013:228:460-5.

24. Melo X, Santa-Clara H, Pimenta NM, Carrolo M, Martins SS, Minderico CS, et al. Body composition phenotypes and carotid intima-media thickness in 11-13-year-old children. Eur J Pediatr. 2014;173:345-52.

25. Osiniri I, Sitjar C, Soriano-Rodriguez P, Prats-Puig A, Casas-Satre C, Mayol L, et al. Carotid intima-media thickness at 7 years of age: relationship to C-reactive protein rather than adiposity. J Pediatr. 2012;160:276-80. e271.

26. Sass C, Herbeth B, Chapet O, Siest G, Visvikis S, Zannad F. Intima-media thickness and diameter of carotid and femoral arteries in children, adolescents and adults from the Stanislas cohort: effect of age, sex, anthropometry and blood pressure. J Hypertens. 1998;16:1593-602.

27. Weghuber D, Zelzer S, Stelzer I, Paulmichl K, Kammerhofer D, Schnedl W, et al. High risk vs"metabolically healthyo" phenotype in juvenile obesity - Neck subcutaneous adipose tissue and serum uric acid are clinically relevant. Exp Clin Endocrinol Diabetes. 2013;121:384-90.

28. Whincup PH, Nightingale CM, Rapala A, Joysurry D, Prescott M, Donald AE, et al. Ethnic differences in carotid intimal medial thickness and carotid-femoral pulse wave velocity are present in UK children. Heart. 2011;97:A41.

29. Croymans DM, Sanchez A, Barth JD, Roberts CK. Carotid intima-media thickness, dietary intake, and cardiovascular phenotypes in adolescents: relation to metabolic syndrome. Metab Clin Exp. 2010;59:533-9.

30. Dawson JD, Sonka M, Blecha MB, Lin W, Davis PH. Risk factors associated with aortic and carotid intima-media thickness in adolescents and young AdultsThe Muscatine offspring study. J Am Coll Cardiol. 2009;53:2273-9.

31. Mittelman SD, Gilsanz P, Mo AO, Wood J, Dorey F, Gilsanz V. Adiposity predicts carotid intima-media thickness in healthy children and adolescents. J Pediatr. 2010;156:592-7. e592.

32. Urbina EM, Kimball TR, McCoy CE, Khoury PR, Daniels SR, Dolan LM. Youth with obesity and obesity-related type 2 diabetes mellitus demonstrate abnormalities in carotid structure and function. Circulation. 2009;119:2913-9.

33. Elkiran O, Yilmaz E, Koc M, Kamanli A, Ustundag B, Ilhan N. The association between intima media thickness, central obesity and diastolic blood pressure in obese and owerweight children: a cross-sectional school-based study. Int J Cardiol. 2013;165:528-32.

34. Ozguven I, Ersoy B, Ozguven A, Ozkol M, Onur E. Factors affecting carotid intima media thickness predicts early atherosclerosis in overweight and obese adolescents. Obesity Res Clin Pract. 2010;4:e41-8.

35. Arnaiz P, Acevedo M, Barja S, Aglony M, Guzman B, Cassis B, et al. Adiponectin levels, cardiometabolic risk factors and markers of subclinical atherosclerosis in children. Int J Cardiol. 2010;138:138-44.

36. Pandit D, Kinare A, Chiplonkar S, Khadilkar A, Khadilkar V. Carotid arterial stiffness in overweight and obese Indian children. J Pediatr Endocrinol Metab. 2011;24:97-102.

37. Lim SM, Kim HC, Lee HS, Lee JY, Suh M, Ahn SV. Association between blood pressure and carotid intima-media thickness. J Pediatr. 2009;154:667-71. e661.

38. Fraser GE. Diet, life expectancy, and chronic disease: studies of seventh-Day adventists and other vegetarians. New York: Oxford University Press; 2003.
39. McGill Jr HC, McMahan CA, Herderick EE, Malcom GT, Tracy RE, Strong JP. Origin of atherosclerosis in childhood and adolescence. Am J Clin Nutr. 2000;72:1307S-15S

40. Scuteri A, Najjar SS, Muller DC, Andres R, Hougaku H, Metter EJ, et al. Metabolic syndrome amplifies the age-associated increases in vascular thickness and stiffness. J Am Coll Cardiol. 2004;43:1388-95.

41. Reis JP, Loria CM, Lewis CE, Powell-Wiley TM, Wei GS, Carr JJ, et al. Association between duration of overall and abdominal obesity beginning in young adulthood and coronary artery calcification in middle age. JAMA. 2013;310:280-8.

42. Urbina E. Noninvasive assessment of target organ injury in children with the metabolic syndrome. J Cardiometab Syndr. 2006;1:277-81.

43. Giannini C, de Giorgis T, Scarinci A, Cataldo I, Marcovecchio ML, Chiarelli F, et al. Increased carotid intima-media thickness in pre-pubertal children with constitutional leanness and severe obesity: the speculative role of insulin sensitivity, oxidant status, and chronic inflammation. Eur. 2009;161:73-80.

44. Iannuzzi A, Licenziati MR, Acampora C, Salvatore V, Auriemma L, Romano $M L$, et al. Increased carotid intima-media thickness and stiffness in obese children. Diabetes Care. 2004:27:2506-8.

\section{Submit your next manuscript to BioMed Central and take full advantage of:}

- Convenient online submission

- Thorough peer review

- No space constraints or color figure charges

- Immediate publication on acceptance

- Inclusion in PubMed, CAS, Scopus and Google Scholar

- Research which is freely available for redistribution 